\title{
Veranstaltungen
}

http://doi.org/10.1515/bd-2018-0107

\section{SYMPOSIUM an der TH Köln}

\section{Mit MALIS Zukunft gestalten - Konzepte und Strategien für die bibliothekarische Praxis}

Anlässlich des 10-jährigen Bestehens des berufsbegleitenden Weiterbildungsstudiengangs Bibliotheks- und Informationswissenschaft (Master in Library and Information Science / MALIS; http://www.malis.th-koeln.de) am Institut für Informationswissenschaft der TH Köln, veranstaltet das MALIS-Team am 31. Januar und 1. Februar 2019 ein Symposium mit namhaften Expert*innen aus der Bibliotheks- und Informationswissenschaft.

Das Programm sowie weitere Informationen zum Symposium sind auf der Veranstaltungswebsite zu finden:

https://www.th-koeln.de/studium/bibliotheks--und-informationswissenschaftmaster-in-library-and-information-science---symposium-2019_58694.php

Das Symposium findet im Gebäude Claudiusstraße 1 der TH Köln statt. Die Veranstaltung ist offen für alle Interessierten!

Die Anmeldung mittels Onlineformular auf der Veranstaltungswebsite ist ab sofort möglich.

Der viersemestrige berufsbegleitende Weiterbildungsstudiengang MALIS (Master in Library and Information Science) qualifiziert seit 2009 Absolvent^innen verschiedener Fachdisziplinen sowie Bibliothekar^innen (Diplom/Bachelor) für Leitungs- und Führungsaufgaben in Bibliotheken und anderen Informationseinrichtungen.

Sein spezifisches Profil gewinnt der Studiengang durch die enge Zusammenarbeit mit der bibliothekarischen und informationswissenschaftlichen Forschung und Praxis. Lehrende sind neben den Mitgliedern des Instituts für Informationswissenschaft der TH Köln Expertinnen aus der Praxis, u. a. Leiter`innen von Bibliotheken und anderen Informationseinrichtungen. 
Die enge Kooperation zwischen Hochschule und Praxis gewährleistet die hohe Aktualität und praktische Relevanz der Studieninhalte. Gleichzeitig ermöglichen frei wählbare Studienschwerpunkte eine an individuellen Interessen orientierte Gestaltung des Studiums. Die Blockstruktur der Präsenzlehrveranstaltungen in Kombination mit E-Learning-Modulen macht zudem eine flexible Organisation des Studiums möglich.

Prof. Dr. Achim Oßwald

TH Köln / Technology, Arts, Sciences

Institut für Informationswissenschaft

Fakultät für Informations- und Kommunikationswissenschaften

Gustav-Heinemann-Ufer 54; 50968 Köln

Tel. + 49 / 221 / 8275-3375

achim.osswald@th-koeln.de 